\title{
Coexistence of catamenial pneumothorax and catamenial hemoptysis in a patient with pulmonary hemangiomatosis-like foci: A case report
}

\author{
Tomohito Saito, MD, ${ }^{\mathrm{a}}$ Tomohiro Maniwa, MD, ${ }^{\mathrm{a}}$ Hiroyuki Kaneda, MD, ${ }^{\mathrm{a}}$ Ken-ichiro Minami, MD, \\ Noriko Sakaida, MD, ${ }^{\mathrm{b}}$ Yoshiko Uemura, MD, ${ }^{\mathrm{b}}$ Akiharu Okamura, MD, ${ }^{\mathrm{c}}$ and Yukihito Saito, MD, PhD, ${ }^{\mathrm{a}}$ \\ Moriguchi, Osaka, and Kakogawa, Hyogo, Japan
}

Catamenial pneumothorax and catamenial hemoptysis are syndromes specific to women of reproductive age. Thoracic endometriosis syndrome most commonly presents as catamenial pneumothorax and very rarely as catamenial hemoptysis. Thoracic endometriosis syndrome is not the only cause of catamenial pneumothorax and hemoptysis. Some cases of catamenial pneumothorax and more than half of patients with hemoptysis lack evidence of ectopic endometrial tissue. Coexistence of catamenial pneumothorax and hemoptysis is extremely rare, and only one patient has been reported in the English literature. ${ }^{1}$ We report a case of catamenial pneumothorax and hemoptysis presenting with pulmonary capillary hemangiomatosis-like foci instead of ectopic endometrial implants.

\section{CLINICAL SUMMARY}

A 19 -year-old woman, gravida 0 para 0 , with a past medical history of recurrent right-sided chest pain and hemoptysis was referred to our hospital for further treatment of her third episode of right-sided pneumothorax. She had already received a thoracostomy, but the air had not stopped bubbling from her chest tube. She remembered the onset of the chest pain was her third day of menstruation. During the previous 9 months, she had been experiencing intermittent hemoptysis every month, which often coincided with menstruation. She never received pelvic surgery or oral contraceptive treatment. She had experienced several episodes of syncope due to orthostatic disturbance.

On admission, computed tomography of the thorax revealed multiple nodules of demarcated consolidation in the upper lobe of the right lung and the lower lobe of the left

\footnotetext{
From the Division of Thoracic and Cardiovascular Surgery, Division of Thoracic Surgery, ${ }^{\mathrm{a}}$ and Department of Surgical Pathology, ${ }^{\mathrm{b}}$ Kansai Medical University, Moriguchi, Osaka, Japan, and Department of Pathology, ${ }^{\mathrm{c}}$ Kakogawa Municipal Hospital, Kakogawa, Hyogo, Japan.

Disclosures: None.

Received for publication May 20, 2008; accepted for publication July 17, 2008; available ahead of print Oct 13, 2008.

Address for reprints: Tomohito Saito, MD, Kansai Medical University, Division of Thoracic Surgery, Department of Thoracic and Cardiovascular Surgery, 10-15 Fumizonocho, Moriguchi, Osaka 570-8507, 573-1191, Japan (E-mail: saitotom@ hirakata.kmu.ac.jp).

J Thorac Cardiovasc Surg 2010;139:e14-6

0022-5223/\$36.00

Crown Copyright (c) 2010 by The American Association for Thoracic Surgery doi:10.1016/j.jtcvs.2008.07.025
}

lung, which had not been detected 6 months before (Figure 1, $A, B)$. During the same interval, a cystic lesion in the upper lobe of the right lung, which had been seen in the previous images, became no longer detectable (Figure 1,B). There was no evidence of peritoneal endometriosis, and the serum level of cancer antigen 125 (CA 125) was $35 \mathrm{U} / \mathrm{mL}$. Systolic pulmonary artery pressure was estimated as $31.3 \mathrm{~mm} \mathrm{Hg}$ by ultrasonographic study. Anti-human immunodeficiency virus antibody was negative.

We suspected she had catamenial hemoptysis and catamenial pneumothorax, and we performed video-assisted thoracoscopic surgery 14 days after the onset of menses. By thoracoscopy, we saw a blue-brown dimpling with bullae on the upper lobe (Figure 2,A) of the right lung. There were a number of red-brown spots on the surface of the lung and emphysematous bullae in the interlobar fissure. The diaphragm appeared normal. We resected the bluebrown lesion with surrounding lung tissue and plicated the necks of the bullae. We put a polyglycolic acid sheet on the stapler line and the diaphragm to prevent recurrent pneumothorax.

Pathologic investigation revealed subpleural hematoma with hemosiderin-laden macrophages. The alveoli were emphysematous, and the interstitium was thickened. There was no evidence of ectopic endometrial implants. Immunohistochemical stains for CD10, estrogen receptor, and progesterone receptor were negative. The lesion consisted of dilated capillaries that had epithelial proliferation without proliferation of smooth muscle cells. The proliferating cells were immunoreactive for CD31 and $\alpha$-smooth muscle actin and negative for melanocyte marker HMB 45 and endothelial marker D2 40. These changes are similar to those seen in pulmonary capillary hemangiomatosis.

The patient was discharged without any medication because she did not want to undergo hormonal therapy with gonadotropin-releasing hormone analogues, which induces menopause. In follow-up, 4 months after the operation, the left-sided pneumothorax and hemoptysis recurred with menstruation. Computed tomography of the chest showed a new cavity-like nodule and ground-glass opacity in the lower lobe of the left lung (Figure 1,C). After that, she agreed to and received gonadotropin-releasing hormone analogue therapy (leuprorelin acetate $1.88 \mathrm{mg}$ intramuscularly per month) for 6 months, during which her left-sided pneumothorax recurred 4 times. 


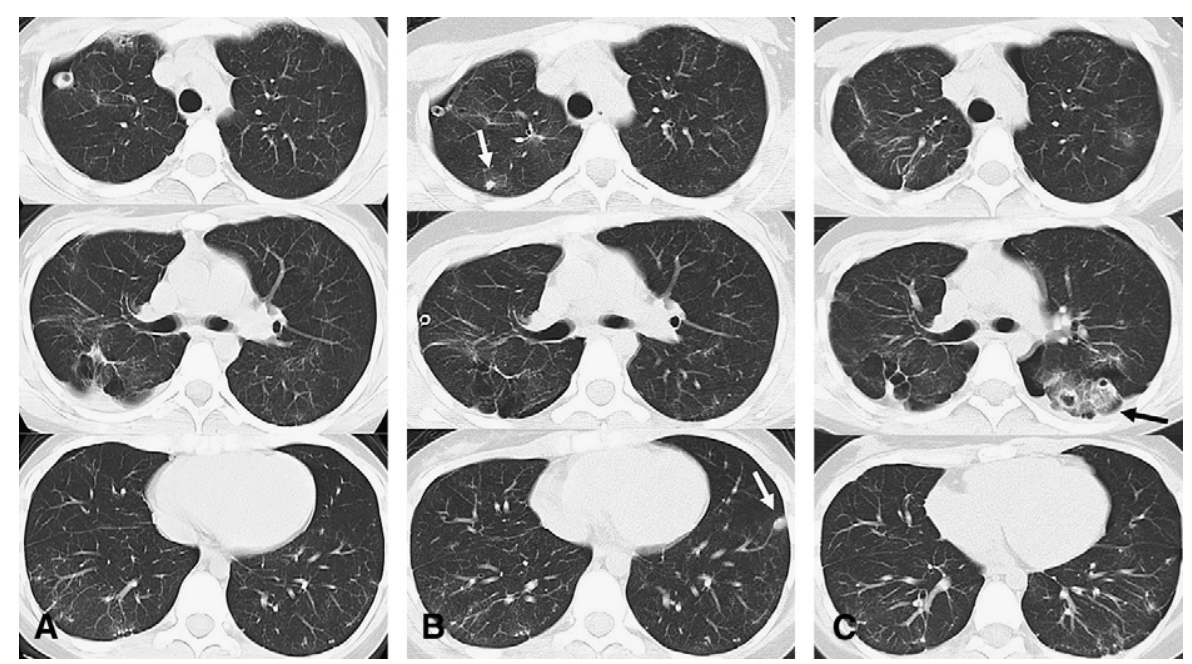

FIGURE 1. Findings of computed tomography of the thorax. Images at 6 months before admission (A) and at admission (B). Micronodules with demarcated consolidation are evident in the right upper lobe and left lower lobe (white arrow, note that a chest tube was inserted). Six months after admission (C), a cavity-like lesion with multiple consolidative spots emerged in the lower lobe of the left lung (black arrow).

\section{DISCUSSION}

We report a very rare case of coexisting bilateral catamenial pneumothorax and hemoptysis. Catamenial hemoptysis is defined as a cyclical hemoptysis occurring during menses and is an extremely rare condition in women; approximately 40 cases have been reported in the English language litera- ture since the first description in $1956 .^{2}$ All these cases were attributed to pulmonary endometriosis, but that was histopathologically confirmed in only one third of the cases. ${ }^{2}$

Catamenial pneumothorax is generally defined as recurrent pneumothorax occurring within 72 hours after the onset of menses. Recently, Alifano and colleagues $^{3}$ showed that
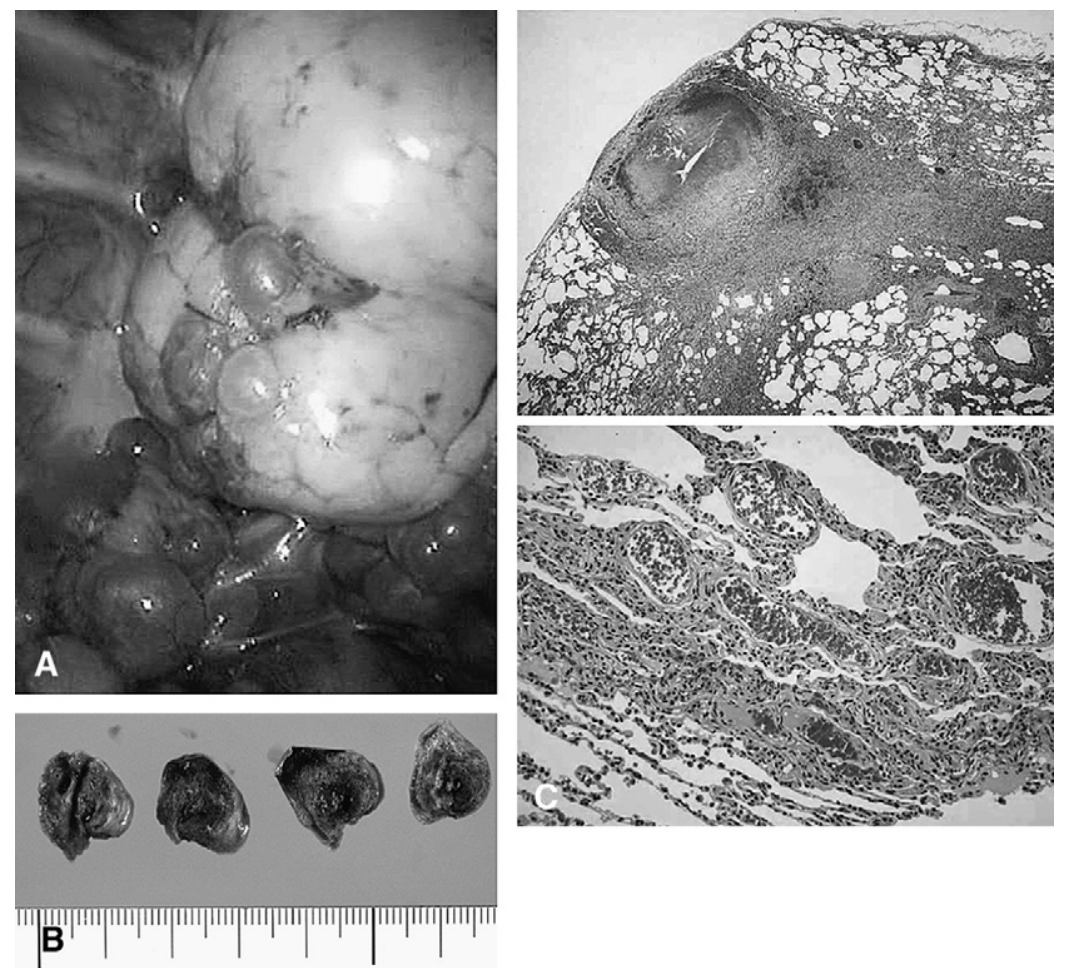

FIGURE 2. (A) Intraoperative endoscopic findings: A blue-brown spot with bullae. Bullae were also observed in the interlobar fissure. (B) Gross photograph of cut surface of resected lung shows an intrapulmonary hematoma. (C) Pathological findings of the resected lung: Intrapulmonary hemorrhage with vascular proliferation There were no ectopic endometrial implants. (Low magnification, and high magnification, hematoxylin and eosin.) 
catamenial pneumothorax accounted for 28 (24.6\%, rightsided in all but one) of 114 women who underwent videoassisted thoracoscopic surgery for spontaneous pneumothorax. Histopathologic examination found endometriosis, mainly diaphragmatic, in 18 patients. Isolated visceral pleural endometriosis was identified in only 1 case. ${ }^{3}$ At our institution, catamenial pneumothorax accounted for $12(21.8 \%)$ of 55 women referred for surgery to address spontaneous pneumothorax from 1990 to 2007. Among these, there was 1 more case with catamenial pneumothorax in which pulmonary capillary hemoangiomatosis-like angioproliferation was found in the resected lung. That patient experienced 4 episodes of right-sided pneumothorax and finally received talc pleurodesis.

Thoracic endometriosis syndrome is characterized by the presence within the thoracic cavity of morphologically normal endometrial tissue, which consists of glands and stroma. Immunohistochemically, most glands and half of the stromal cells show intense nuclear staining for estrogen and progesterone receptors. ${ }^{4} \mathrm{CD} 10$ positivity is also helpful to confirm the presence of endometrial stroma. The presented case lacked CD10 positivity and any other evidence of ectopic endometrial implants. Even in patients with thoracic endometriosis syndrome, histopathologic confirmation of pulmonary endometriosis is impossible when the resection of the lung is performed during the menstrual period. Our patient received her operation 13 days after the onset of her menses, but only pulmonary capillary hemangiomatosis-like foci were identified in the resected tissue. A second pathologist confirmed the pathologic diagnosis as pulmonary capillary hemangiomatosis-like foci, without any evidence of endometriosis. Preoperative serum level of CA 125 is a useful predictor of thoracic endometriosis syndrome. ${ }^{5}$ In our case, it was lower than the cutoff level of $39 \mathrm{U} / \mathrm{mL}$, which indicated an etiology other than thoracic endometriosis syndrome.

Pulmonary capillary hemangiomatosis is a rare cause of primary pulmonary hypertension. Although it is characteristically associated with primary pulmonary hypertension in young patients, Havlik and colleagues ${ }^{6}$ have reported 8 autopsy cases with pulmonary capillary hemangiomatosislike foci that were found incidentally. Also, Umezu and colleagues $^{7}$ have reported a case of pulmonary capillary hemangiomatosis without evidence of primary pulmonary hypertension. Reportedly, hemoptysis is seen in more than $30 \%$ of cases with pulmonary capillary hemangiomatosis. Several reports show a possible association between estrogen and hemangiomatosis or vascular proliferation.
Elevation of serum estrogen levels promoted a systemic increase in host angiogenesis in patients with estrogen receptor-negative breast cancers. ${ }^{8}$ Ozakyol and Kebapci ${ }^{9}$ reported that estrogen replacement therapy enhanced the growth of hemangiomatosis in the liver in 2 patients. Given these facts, catamenial pneumothorax and hemoptysis in this case might be the result of vascular proliferation and recurrent hemorrhage in the lungs due to an abnormal response to circulating estrogen.

We had launched our initial treatment of this patient with hormonal therapy, which is usually used for endometriosis, because we thought the etiology was the same as endometriosis despite the different pathologic presentation. However, this patient's postoperative gonadotropin-releasing hormone analogue treatment was not effective, resulting in persistently recurrent catamenial pneumothorax and hemoptysis. Radiologic imaging showed spread of the disease. This fact suggested that catamenial pneumothorax or hemoptysis had another etiology, which was essentially refractory to hormonal therapy. We may consider talc pleurodesis as well as lung transplantation if the disease continues to progress.

We would like to express our gratitude to Dr Tadaaki Hiruki for English editing of this article.

\section{References}

1. Parker CM, Nolan R, Lougheed MD. Catamenial hemoptysis and pneumothorax in a patient with cystic fibrosis. Can Respir J. 2007;14:295-7.

2. Inoue T, Kurokawa Y, Kaiwa Y, Abo M, Takayama T, Ansai M, et al. Videoassisted thoracoscopic surgery for catamenial hemoptysis. Chest. 2001;120: 655-8.

3. Alifano M, Jablonski C, Kadiri H, Falcoz P, Gompel A, Camilleri-Broet S, et al. Catamenial and non-catamenial, endometriosis-related or not pneumothorax referred for surgery. Am J Respir Crit Care Med. 2007;176:1048-53.

4. Fleider DB, Moran CA, Travis WD, Koss MN, Mark EJ. Pleuro-pulmonary endometriosis and pulmonary deciduosis: a clinicopathologic and immunohistochemical study of 10 cases with emphasis on diagnostic pitfalls. Hum Pathol. 1998;29: 1495-503.

5. Bagan P, Berna P, Assouad J, Hupertan V, Le Pimpec Barthes F, Riquet M. Value of cancer antigen 125 for diagnosis of pleural endometriosis in females with recurrent pneumothorax. Eur Respir J. 2008;31:140-2.

6. Havlik DM, Massie LW, Williams WL, Crooks LA. Pulmonary capillary hemangiomatosis-like foci: an autopsy study of 8 cases. Am J Clin Pathol. 2000;113: 655-62.

7. Umezu H, Naito M, Yagisawa K, Hattori A, Aizawa Y. An autopsy case of pulmonary capillary hemangromatosis without evidence of pulmonary hypertension. Virchows Arch. 2001;439:586-92.

8. Gupta PB, Proia D, Cingoz O, Weremowicz J, Naber SP, Weinberg RA, et al. Systemic stromal effects of estrogen promote the growth of estrogen receptor-negative cancers. Cancer Res. 2007;67:2062-71.

9. Ozakyol A, Kebapci M. Enhanced growth of hepatic hemangiomatosis in two adults after postmenopausal estrogen replacement therapy. Tohoku J Exp Med. 2006;210:257-61. 